Research Article

\title{
Bending of a Viscoelastic Timoshenko Cracked Beam Based on Equivalent Viscoelastic Spring Models
}

\author{
Chao Fu $\mathbb{D D}^{1}$ and Xiao Yang ${ }^{2}$ \\ ${ }^{1}$ College of Architecture and Civil Engineering, Xinyang Normal University, Xinyang 464000, China \\ ${ }^{2}$ Scientific Bureau, Shanghai Customs College, Shanghai 201204, China \\ Correspondence should be addressed to Chao Fu; jerryfc@shu.edu.cn
}

Received 9 August 2021; Accepted 11 October 2021; Published 21 October 2021

Academic Editor: Piotr Smarzewski

Copyright ( $) 2021$ Chao Fu and Xiao Yang. This is an open access article distributed under the Creative Commons Attribution License, which permits unrestricted use, distribution, and reproduction in any medium, provided the original work is properly cited.

\begin{abstract}
Considering the transverse crack as a massless viscoelastic rotational spring, the equivalent stiffness of the viscoelastic cracked beam is derived by Laplace transform and the generalized Dirac delta function. Using the standard linear solid constitutive equation and the inverse Laplace transform, the analytical expressions of the deflection and rotation angle of the viscoelastic Timoshenko beam with an arbitrary number of open cracks are obtained in the time domain. By numerical examples, the bending results of the analytical expressions are verified with those of the FEM program. Additionally, the effects of the time, slenderness ratio, and crack depth on the bending deformations of the different cracked beam models are revealed.
\end{abstract}

\section{Introduction}

Viscoelastic materials [1,2] are widely used in civil, mechanical, biological, and aerospace engineering. Since the 1950 s, several research works on the static bending deformations [3, 4], stability [5-7], and dynamic characteristics $[8,9]$ of linear viscoelastic materials were performed by the correspondence principle, integral transformation, finite element method (FEM), differential quadrature method $[10,11]$, and other numerical methods $[12,13]$.

Considering the influences of the external loads, environment, and self-defects, there are some subtle or significant cracks in the structural members during their service life, which leads to the reduction in the bearing capacity of the structure and poses a serious threat to its normal use. Thus, many literatures focused on the research of crack effect and crack identification. Ding et al. [14] and Caddemi et al. [15] pointed out that the equivalent rotational spring model of cracks can be used to analyze the mechanical properties of cracked beams. Biondi and Caddemi [16] proposed that multiple singularities in the flexural stiffness can be used to represent the effect of the concentrated damage of the cracked beam, and the Dirac delta function is proper to represent the singular flexural stiffness corresponding to the crack position. The negative Dirac delta function is employed to present the damage of the flexural stiffness at the crack location by Buda and Caddemi [17] and Caddemi and Calió [18]; however, the flexural stiffness cannot be negative, so this description is physically imperfect. Then, Palmeri and Cicirello [19] and Cicirello and Palmeri [20] presented a physically based "flexibility modelling" of concentrated damage to avoid physical incompleteness. Similarly, by considering the effect of crack gap, Yang et al. [21] gave the equivalent flexural rigidity using Dirac delta function and present the closed form general solutions of bending deformation of Timoshenko beams with an arbitrary number of cracks. Donà et al. [22] developed a twonode multidamaged beam element to analyze the static and dynamic behaviors of the cracked beam, which is suitable for the beam with axial spring, rotational spring, and shearing spring.

It is worth noticing that a large number of articles focused on the mechanical properties of elastic cracked beams, but only a few were concerned about the effects of defects or cracks on the mechanical properties of viscoelastic beams and columns. Stevens and Evan-Iwanowski [23] studied the 
effect of initial curvature defects on the dynamic stability of the viscoelastic column under a periodic axial force by the complex modulus method. Considering the initial lateral deflections as imperfections, Szyszkowski and Glockner [24] analyzed the dynamic and static behaviors of the columns made of a linear three-element model material. According to the Boltzmann superposition linear viscoelasticity, Touati and Cederbaum [25] studied the postbuckling behavior of imperfect columns made of linear viscoelastic materials. By the Extended Finite Element Method and generalized Heaviside function, Zhang et al. [26] numerically investigated the deformations in a viscoelastic cracked body such as crack opening and sliding displacements. Combining the Galerkin method and multiple scale method, Younesian et al. [27] analyzed the frequency response of a cracked beam supported by a nonlinear viscoelastic foundation. Based on the Fourier transform and the principle of virtual work, Sarvestan et al. [28] presented a spectral finite element model for vibration analysis of the Euler-Bernoulli Kelvin-Voigt cracked beam.

Generally, there are three categories for the modeling of cracks in beam structures [14]: local stiffness reduction, discrete spring model, and Finite Element Method. Considering that this approach suffers from in matching the quantitative relationship between crack depth and damage severity, only a few literatures used local stiffness reduction method to analyze the effect of cracks. Additionally, for the expensively computational cost, 2D and 3D complex Finite Element Methods are mostly carried out to describe the mechanical behaviors of the beam with a breathing crack model or nonlinear dynamic problems. Therefore, a lot of literatures preferred to use the discrete spring model to study the effects of crack depth, crack location, and crack number on the dynamic and static characteristics of the cracked beams. However, up to now, most literatures focused on elastic cracked beams or viscoelastic intact (nondamaged) beams, and only a few studies were performed on the mechanical behaviors of viscoelastic cracked beam structures. In addition, when there is a large gap in the structure or component without closure, the open crack model is very suitable. Considering the material properties of the beam, the crack is equivalent to a viscoelastic rotational spring, which can describe the crack effect more reasonably. Using the generalized functions and mechanical compatibility conditions at the crack position, the general explicit analytical expressions for bending of a viscoelastic Timoshenko beam having an arbitrary number of open cracks are derived to analyze the static behavior of the viscoelastic cracked beam more conveniently and accurately.

Therefore, regarding the transversal crack as a massless viscoelastic rotational spring, the equivalent bending stiffness of the viscoelastic cracked beam with open cracks is presented in Laplace domain. Then, the general explicit analytical expressions in time domain for the deflection and rotation angle of a Timoshenko beam having an arbitrary number of open cracks and satisfying the standard linear solid constitutive equation of viscoelasticity are derived. Also, the finite element method is applied to verify the present expressions. By numerical examples, the effects of the time, slenderness ratio, and crack depth on the bending deformation under the simply supported boundary condition on different cracked beam models are analyzed. Additionally, by comparing with the results of Euler-Bernoulli cracked beam, the influence of transversal shearing deformation on the Timoshenko cracked beam is revealed.

\section{Methods}

2.1. Equivalent Bending Stiffness of a Viscoelastic Cracked Beam. According to the constitutive equation of the standard linear solid [29], the physical model of the viscoelastic beam is given as Figure 1. Assuming $E_{1}$ and $E_{2}$ as the elastic moduli of an elastic element, and $\eta$ as the viscosity coefficient of a viscous element, the relaxation modulus $Y(t)$ and shear modulus $G(t)$ are defined as follows:

$$
\begin{aligned}
& Y(t)=q_{0}+\left(\frac{q_{1}}{p_{1}}-q_{0}\right) e^{-t / p_{1}}, \\
& G(t)=\frac{Y(t)}{2(1+v)}
\end{aligned}
$$

where the Poisson's ratio $v$ is a constant, and

$$
\begin{aligned}
& p_{1}=\frac{\eta}{E_{1}+E_{2}}, \\
& q_{0}=\frac{E_{1} E_{2}}{E_{1}+E_{2}}, \\
& q_{1}=\frac{E_{1} \eta}{E_{1}+E_{2}} .
\end{aligned}
$$

2.1.1. Transverse Bending Moment and Shear Force. As shown in Figure 2, there is a viscoelastic rectangular beam of length $L$, height $h$, and width $b$ in the coordinate axis $x$, $y$, and $z$, respectively, which has a transverse open crack $j(j=1,2, \ldots, N)$ with crack depth $\mathrm{d}_{j}$ at the coordinate $x=x_{j}$, respectively. Let us consider that $w(x, t)$ and $\varphi(x, t)$ are the transverse deflection of the axial line and the rotation angle of the beam cross section subjected to the distributed transverse load $q(x, t)$. According to the hypothesis of the Timoshenko beam theory, the displacements $u_{1}(x, y, z, t)$ and $u_{2}(x, y, z, t)$ in the $x$ and $y$ directions, respectively, can be expressed as follows:

$$
\begin{aligned}
& u_{1}(x, y, z, t)=-y \varphi(x, t), \\
& u_{2}(x, y, z, t)=w(x, t) .
\end{aligned}
$$

The axial normal strain and shear strain of the cross section are given as

$$
\begin{aligned}
& \varepsilon(x, y, z, t)=-y \frac{\partial \varphi(x, t)}{\partial x} \\
& \gamma(x, y, z, t)=-\varphi(x, t)+\frac{\partial w(x, t)}{\partial x} .
\end{aligned}
$$




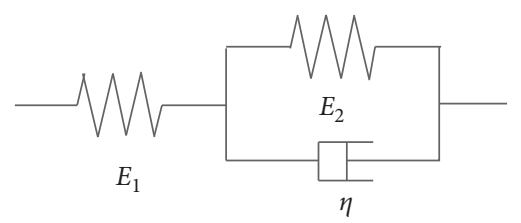

Figure 1: Physical model of the standard linear solid constitutive relation of viscoelasticity.

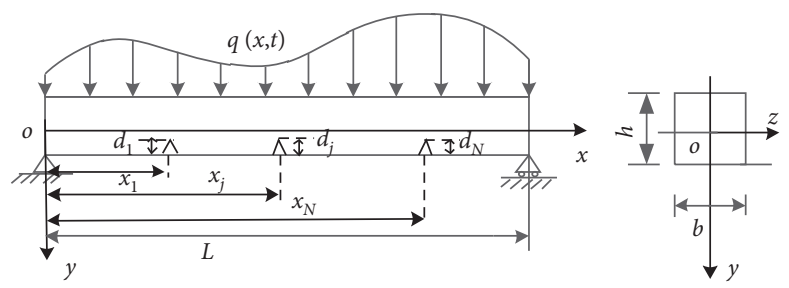

Figure 2: Geometric parameters of a viscoelastic Timoshenko cracked beam.

The axial normal stress and shear stress of the beam cross section are written as follows:

$$
\begin{aligned}
& \sigma(x, y, z, t)=Y(0) \mathcal{\varepsilon}(x, t)+\dot{Y}(t) * \varepsilon(x, t), \\
& \tau(x, y, z, t)=G(0) \gamma(x, t)+\dot{G}(t) * \gamma(x, t),
\end{aligned}
$$

where $Y(t)$ is the first derivative of $Y(t)$ with respect to the time $t$, and the asterisk * denotes the convolution, i.e. $f(t)^{*} g(t)=\int_{0}^{t} f(\tau) g(t-\tau) \mathrm{d} \tau$.

The bending moment $M(x, t)$ and shearing force $F_{\mathrm{s}}(x, t)$ on the beam cross section are given as

$$
\left\{\begin{array}{l}
M(x, t)=-I\left[Y(0) \frac{\partial \varphi(x, t)}{\partial x}+\dot{Y}(t) * \frac{\partial \varphi(x, t)}{\partial x}\right], \\
F_{s}(x, t)=\kappa A\left\{G(0)\left[-\varphi(x, t)+\frac{\partial w(x, t)}{\partial x}\right]+\dot{G}(t) *\left[-\varphi(x, t)+\frac{\partial w(x, t)}{\partial x}\right]\right\},
\end{array}\right.
$$

where $\kappa$ is the shear correction factor of the Timoshenko beam. The cross-section $A$ and moment of inertia of the neutral axis $I$ are given as

$$
I=\iint_{\Omega} y^{2} \mathrm{~d} y \mathrm{~d} z, A=\iint_{\Omega} \mathrm{d} y \mathrm{~d} z .
$$

Denote the Laplace transform of the bending moment $M(x, t)$ and shearing force $F_{s}(x, t)$ by $\bar{M}(x, s)$ and $\bar{F}_{s}(x, s)$,

$$
\begin{aligned}
& \bar{M}(x, s)=-s \bar{Y}(s) I \frac{\partial \bar{\varphi}(x, s)}{\partial x}, \\
& \bar{F}_{s}(x, s)=s \bar{G}(s) \kappa A\left[-\bar{\varphi}(x, s)+\frac{\partial \bar{w}(x, s)}{\partial x}\right] .
\end{aligned}
$$

Obviously, $s \bar{Y}(s) I$ and $s \bar{G}(s) \kappa A$ are the bending stiffness and shear stiffness of the viscoelastic intact beam in Laplace domain. The superscript - denotes the Laplace transform of the function with respect to the time $t$, and $s$ is the Laplace transform parameter.

Thus, the axial bending curvature of the viscoelastic beam in Laplace domain is given as

$$
\frac{\partial \bar{\varphi}(x, s)}{\partial x}=-\frac{\bar{M}(x, s)}{s \bar{Y}(s) I}
$$

2.1.2. Equivalent Bending Stiffness of the Viscoelastic Beam with Open Cracks. Assuming that the crack at the location $x=x_{j}(j=1,2, \ldots, N)$ is always open, and it can be equivalent as a massless viscoelastic rotational spring. Let us denote the bending moment of the crack $j$ at $x=x_{j}$ by $M_{j}(t)$, and the equivalent stiffness of the viscoelastic rotational spring of the crack by $k_{j}(t)$, respectively. The rotation angle $\Delta_{j}(t)$ of the equivalent rotational spring can be expressed as

$$
M_{j}(t)=-\left[k_{j}(0) \Delta_{j}(t)+\dot{k}_{j}(t) * \Delta_{j}(t)\right] .
$$

By Laplace transform, we have

$$
\bar{M}_{j}(s)=-s \bar{k}_{j}(s) \bar{\Delta}_{j}(s) .
$$

The rotation angle of the equivalent rotational spring at the open crack $j$ in Laplace domain is 


$$
\bar{\Delta}_{j}(s)=-\frac{\bar{M}_{j}(s)}{s \bar{k}_{j}(s)} .
$$

Based on the crack effect and Laplace transform, the rotation angle of beam cross section in time domain and Laplace domain can be expressed as, respectively.

$$
\begin{aligned}
& \phi(x, t)=\varphi(x, t)+\sum_{j=1}^{N} \Delta_{j}(t) H\left(x-x_{j}\right), \\
& \bar{\phi}(x, s)=\bar{\varphi}(x, s)+\sum_{j=1}^{N} \bar{\Delta}_{j}(s) H\left(x-x_{j}\right),
\end{aligned}
$$

where $H(x)$ is the Heaviside function [21].

The axial bending curvature of the viscoelastic cracked beam in Laplace domain is rewritten as

$$
\begin{aligned}
\frac{\partial \bar{\phi}(x, s)}{\partial x} & =\frac{\partial \bar{\varphi}(x, s)}{\partial x}+\sum_{j=1}^{N} \bar{\Delta}_{j}(s) \delta\left(x-x_{j}\right) \\
& =-\frac{\bar{M}(x, s)}{s \bar{Y}(s) I}-\sum_{j=1}^{N} \frac{\bar{M}_{j}(s)}{s \bar{k}_{j}(s)} \delta\left(x-x_{j}\right),
\end{aligned}
$$

where $\delta(x)$ is the Dirac delta function [21].

Denote the equivalent bending stiffness of a viscoelastic beam with open cracks by $(\mathrm{EI})_{\mathrm{e}}(x, t)$. Then, from the formula,

$$
M(x, t)=-\left[(\mathrm{EI})_{e}(x, 0) \frac{\partial \phi(x, t)}{\partial x}+(\dot{\mathrm{EI}})_{e}(x, t) * \frac{\partial \phi(x, t)}{\partial x}\right] .
$$

By Laplace transform, we have

$$
\bar{M}(x, s)=-s(\overline{\mathrm{EI}})_{e}(x, s) \frac{\partial \bar{\phi}(x, s)}{\partial x} .
$$

Combining equations (14) and (16), we obtain

$$
\frac{1}{(\overline{\mathrm{EI}})_{e}(x, s)}=\frac{1}{\bar{Y}(s) I}+\sum_{j=1}^{N} \frac{1}{\bar{k}_{j}(s)} \delta\left(x-x_{j}\right) .
$$

If the beam is made of an elastic material, $Y(t)$ and $k_{j}(t)$ both are constants, i.e., $Y(t)=E, k_{j}(t)=k_{j}$, equation (17) can be rewritten as

$$
(\overline{\mathrm{EI}})_{e}=\left[\frac{1}{\mathrm{EI}}+\sum_{j=1}^{N} \frac{1}{k_{j}} \delta\left(x-x_{j}\right)\right]^{-1} \frac{1}{s} .
$$

By the inverse Laplace transformation, we obtain

$$
(\mathrm{EI})_{\mathrm{e}}=\left[\frac{1}{\mathrm{EI}}+\sum_{j=1}^{N} \frac{1}{k_{j}} \delta\left(x-x_{j}\right)\right]^{-1} .
$$

It is seen that equation (19) is the same expression of the equivalent bending stiffness of the elastic beam with open cracks in the time domain derived by Yang et al. [21].

By equations (6) and (7), the shearing force of the viscoelastic Timoshenko cracked beam can be expressed as

$$
\left\{\begin{array}{l}
F_{s}(x, t)=\kappa \iint_{A}\left\{G(0)\left[-\phi(x, t)+\frac{\partial w(x, t)}{\partial x}\right]+\dot{G}(t) *\left[-\phi(x, t)+\frac{\partial w(x, t)}{\partial x}\right]\right\} \mathrm{d} y \mathrm{~d} z \\
\bar{F}_{s}(x, s)=s \bar{G}(s) \kappa A\left[-\bar{\phi}(x, s)+\frac{\partial \bar{w}(x, s)}{\partial x}\right]
\end{array}\right.
$$

2.1.3. Bending Deformation of the Viscoelastic Cracked Beam. The governing equations are given as

$$
\begin{gathered}
\frac{\partial F_{s}(x, t)}{\partial x}+q(x, t)=0, \\
\frac{\partial M(x, t)}{\partial x}-F_{s}(x, t)=0 .
\end{gathered}
$$
have

By Laplace transform and integrating computation, we

$$
\begin{aligned}
& \bar{F}_{s}(x, s)=-\left[\bar{q}^{[1]}(x, s)+C_{1}\right], \\
& \bar{M}(x, s)=-\left[\bar{q}^{[2]}(x, s)+C_{1} x+C_{2}\right],
\end{aligned}
$$

where $C_{1}$ and $C_{2}$ are arbitrary constants, and

$$
\left\{\begin{array}{l}
\bar{q}^{[1]}(x, s)=\int_{0}^{x} \bar{q}\left(t_{1}, s\right) \mathrm{d} t_{1}, \\
\bar{q}^{[3]}(x, s)=\int_{0}^{x} \int_{0}^{t_{3}} \int_{0}^{t_{2}} \bar{q}\left(t_{1}, s\right) \mathrm{d} t_{1} \mathrm{~d} t_{2} \mathrm{~d} t_{3}, \\
\bar{q}^{[2]}(x, s)=\int_{0}^{x} \int_{0}^{t_{2}} \bar{q}\left(t_{1}, s\right) \mathrm{d} t_{1} \mathrm{~d} t_{2}, \\
\bar{q}^{[4]}(x, s)=\int_{0}^{x} \int_{0}^{t_{4}} \int_{0}^{t_{3}} \int_{0}^{t_{2}} \bar{q}\left(t_{1}, s\right) \mathrm{d} t_{1} \mathrm{~d} t_{2} \mathrm{~d} t_{3} \mathrm{~d} t_{4} .
\end{array}\right.
$$

Combining equations (16), (20) and (22), 


$$
\left\{\begin{array}{l}
s \bar{G}(s) \kappa A\left[-\bar{\phi}(x, s)+\frac{\partial \bar{w}(x, s)}{\partial x}\right]=-\left[\bar{q}^{[1]}(x, s)+C_{1}\right], \\
s(\overline{\mathrm{EI}})_{\mathrm{e})}(x, s) \frac{\partial \bar{\phi}(x, s)}{\partial x}=\bar{q}^{[2]}(x, s)+C_{1} x+C_{2} .
\end{array}\right.
$$

Then, we have

$$
\frac{\partial}{\partial x}\left[\frac{\partial \bar{w}(x, s)}{\partial x}\right]=\frac{\bar{q}^{[2]}(x, s)+C_{1} x+C_{2}}{s(\overline{\mathrm{EI}})_{\mathrm{e}}(x, s)}-\frac{\bar{q}(x, s)}{s \bar{G}(s) \kappa A} .
$$

Utilizing the properties of the function $\delta(x)$ and equations (17) and (25) can be rewritten as

$$
\begin{aligned}
\bar{w}(x, s)= & -\frac{1}{s \bar{G}(s) \kappa A} \bar{q}^{[2]}(x, s)+\frac{1}{s \bar{Y}(s) I}\left[\bar{q}^{[4]}(x, s)+\frac{1}{6} C_{1} x^{3}+\frac{1}{2} C_{2} x^{2}\right]+C_{3} x+C_{4} \\
& +\sum_{j=1}^{N} \frac{1}{s \bar{s}_{j}(s)}\left(x-x_{j}\right) H\left(x-x_{j}\right)\left[\bar{q}^{[2]}\left(x_{j}, s\right)+C_{1} x_{j}+C_{2}\right]
\end{aligned}
$$

where $C_{3}$ and $C_{4}$ are arbitrary constants.

From the first formula of equation (24), we have

$$
\begin{aligned}
\bar{\phi}(x, s)= & \frac{1}{s \bar{G}(s) \kappa A} C_{1}+\frac{1}{s \bar{Y}(s) I}\left[\bar{q}^{[3]}(x, s)+\frac{1}{2} C_{1} x^{2}+C_{2} x\right]+C_{3} \\
& +\sum_{j=1}^{N} \frac{1}{s \bar{k}_{j}(s)} H\left(x-x_{j}\right)\left[\bar{q}^{[2]}\left(x_{j}, s\right)+C_{1} x_{j}+C_{2}\right] .
\end{aligned}
$$

Generally, utilizing the boundary conditions of the beam in Laplace domain, there is a system of linear algebraic equations containing undetermined functions $C_{i}=C_{i}(s)(i=$ $1,2,3,4)$, which can be solved. Then, substituting the functions $C_{i}=C_{i}(s)$ into equations (26) and (27), the expressions for the bending deformation of the viscoelastic Timoshenko cracked beam in Laplace domain can be derived.

\subsection{Bending Deformation of the Viscoelastic Timoshenko Cracked Beam Subjected to a Sudden Load}

2.2.1. Bending of a Simply Supported Cracked Beam under a Uniform Sudden Load. Let us consider a simply supported Timoshenko beam with open cracks subjected to a uniform sudden load $q(x, t)=Q_{0} H(t)$. By Laplace transform, equation (1) can be rewritten as

$$
\begin{aligned}
& \bar{Y}(s)=\frac{q_{0}+s_{1}}{s\left(1+s p_{1}\right)}, \\
& \bar{G}(s)=\lambda_{1} \frac{q_{0}+s q_{1}}{s\left(1+s p_{1}\right)},
\end{aligned}
$$

where $\lambda_{1}=0.5(1+\nu)^{-1}$ is the parameter determined by the Poisson's ratio $\nu$.

Based on the equivalent rotational spring model of the crack [20] and Laplace transform, the equivalent spring stiffness at the location $x=x_{j}$ with the crack depth $\mathrm{d}_{j}$ can be expressed as

$$
\begin{aligned}
k_{j}(t) & =\mu_{j} \operatorname{IY}(t), \\
\bar{k}_{j}(s) & =\mu_{j} I \bar{Y}(s), \\
\mu_{j} & =\frac{(0.9 / h)\left[\left(\mathrm{d}_{j} / h\right)-1\right]^{2}}{\left(\mathrm{~d}_{j} / h\right)\left[2-\left(\mathrm{d}_{j} / h\right)\right]} .
\end{aligned}
$$

The parameter $Q_{0}$ is a constant, and

$$
\begin{gathered}
\bar{q}(x, s)=\frac{Q_{0}}{s}, \\
\bar{q}^{[1]}(x, s)=\frac{Q_{0}}{s} x, \\
\bar{q}^{[2]}(x, s)=\frac{Q_{0}}{2 s} x^{2}, \\
\bar{q}^{[3]}(x, s)=\frac{Q_{0}}{6 s} x^{3}, \\
\bar{q}^{[4]}(x, s)=\frac{Q_{0}}{24 s} x^{4} .
\end{gathered}
$$

The boundary conditions of the simply supported Timoshenko beam in Laplace domain are 


$$
\begin{gathered}
\bar{w}(0, s)=0 \\
\bar{w}(L, s)=0, \\
\frac{\partial \bar{\phi}(0, s)}{\partial x}=0 \\
\frac{\partial \bar{\phi}(L, s)}{\partial x}=0 .
\end{gathered}
$$

Then, these four constants are determined as follows:

$$
C_{1}=-\frac{Q_{0} L}{2 s}
$$

$$
\begin{aligned}
& C_{2}=0, \\
& C_{3}=Q_{0}\left[\frac{L}{2 s^{2} \kappa A \bar{G}(s)}+\frac{L^{3}}{24 s^{2} \bar{Y}(s) I}+\sum_{j=1}^{N} \frac{x_{j}\left(L-x_{j}\right)^{2}}{2 \operatorname{Ls}^{2} \bar{k}_{j}(s)}\right], \\
& C_{4}=0 .
\end{aligned}
$$

Substituting equation (32) into equations (22), (26) and (27), the expressions for the deflection, rotation angle, bending moment, and shearing force of the simply supported Timoshenko cracked beam in time domain can be expressed as

$$
\left\{\begin{array}{l}
w(x, t)=\frac{Q_{0}}{2 \kappa A} \lambda_{3}(t) x(L-x)+\frac{Q_{0}}{24 I} \lambda_{2}(t)\left(x^{4}-2 L x^{3}+L^{3} x\right)+\sum_{j=1}^{N} \frac{Q_{0}}{2} \lambda_{4 j}(t) x_{j}\left(L-x_{j}\right)\left[\frac{\left(L-x_{j}\right) x}{L}-\left(x-x_{j}\right) H\left(x-x_{j}\right)\right], \\
\phi(x, t)=\frac{Q_{0}}{24 I} \lambda_{2}(t)\left(4 x^{3}-6 L x^{2}+L^{3}\right)+\sum_{j=1}^{N} \frac{Q_{0}}{2} \lambda_{4 j}(t) x_{j}\left(L-x_{j}\right)\left[\frac{\left(L-x_{j}\right)}{L}-H\left(x-x_{j}\right)\right], \\
M(x, t)=\frac{Q_{0}}{2} x(L-x), \\
F_{s}(x, t)=\frac{Q_{0}}{2}(L-2 x) .
\end{array}\right.
$$

Suppose that $L^{-1}[\cdot]$ is the inverse Laplace transform of the function, and

$$
\left\{\begin{array}{l}
\lambda_{2}(t)=L^{-1}\left[\frac{1}{s^{2} \bar{Y}(s)}\right] \\
=\frac{1}{q_{0}}+\left(p_{1}-\frac{q_{1}}{q_{0}}\right) \frac{1}{q_{1}} \mathrm{e}^{-q_{0} / q_{1} t}, \\
\lambda_{3}(t)=L^{-1}\left[\frac{1}{s^{2} \bar{G}(s)}\right] \\
=\frac{1}{\lambda_{1}}\left[\frac{1}{q_{0}}+\left(p_{1}-\frac{q_{1}}{q_{0}}\right) \frac{1}{q_{1}} \mathrm{e}^{-q_{0} / q_{1} t}\right], \\
\lambda_{4 j}(t)=L^{-1}\left[\frac{1}{s^{2} \bar{k}_{j}(s)}\right] \\
=\frac{1}{\mu_{j} I}\left[\frac{1}{q_{0}}+\left(p_{1}-\frac{q_{1}}{q_{0}}\right) \frac{1}{q_{1}} \mathrm{e}^{-q_{0} / q_{1} t}\right] .
\end{array}\right.
$$


Introduce the following dimensionless variables and parameters

$$
\left\{\begin{array}{l}
t^{*}=\frac{t}{T}, w^{*}=\frac{w}{L}, \phi^{*}=\phi, \xi=\frac{x}{L}, \xi_{j}=\frac{x_{j}}{L}, d_{j}^{*}=\frac{d_{j}}{L}, h^{*}=\frac{h}{L}, I^{*}=\frac{I}{L^{4}}, A^{*}=\frac{A}{L^{2}}, \\
M^{*}=\frac{M}{E_{1} L^{3}}, F_{s}^{*}=\frac{F_{s}}{E_{1} L^{2}}, Q^{*}=\frac{Q_{0}}{E_{1} L}, E_{2}^{*}=\frac{E_{2}}{E_{1}}, \eta_{2}^{*}=\frac{\eta_{2}}{E_{1} T}, p_{1}^{*}=\frac{\eta_{2}^{*}}{1+E_{2}^{*}}, q_{0}^{*}=\frac{E_{2}^{*}}{1+E_{2}^{*}}, \\
q_{1}^{*}=\frac{\eta_{2}^{*}}{1+E_{2}^{*}}, Y^{*}=\frac{Y}{E_{1}}, \mu_{j}^{*}=\mu_{j} L, k_{j}^{*}=Y^{*} I^{*} \mu_{j}^{*}, \lambda_{2}^{*}=E_{1} \lambda_{2}, \lambda_{3}^{*}=E_{1} \lambda_{3}, \lambda_{4 j}^{*}=E_{1} L^{3} \lambda_{4 j},
\end{array}\right.
$$

where $T$ is the time, and it has $T=1 h$.

Thus, the expressions for the deflection, rotation angle, bending moment, and shearing force can be expressed as

$$
\left\{\begin{array}{l}
w^{*}\left(\xi, t^{*}\right)=\frac{\lambda_{3}^{*} Q^{*}}{2 \kappa A^{*}} \xi(1-\xi)+\frac{\lambda_{2}^{*} Q^{*}}{24 I^{*}}\left(\xi^{4}-2 \xi^{3}+\xi\right) \\
+\sum_{j=1}^{N} \frac{\lambda_{4 j}^{*} Q^{*}}{2} \xi_{j}\left(1-\xi_{j}\right)\left[\left(1-\xi_{j}\right) \xi-\left(\xi-\xi_{j}\right) H\left(\xi-\xi_{j}\right)\right], \\
\phi^{*}\left(\xi, t^{*}\right)=\frac{\lambda_{2}^{*} Q^{*}}{24 I^{*}}\left(4 \xi^{3}-6 \xi^{2}+1\right)+\sum_{j=1}^{N} \frac{\lambda_{4 j}^{*} Q^{*}}{2} \xi_{j}\left(1-\xi_{j}\right)\left[\left(1-\xi_{j}\right)-H\left(\xi-\xi_{j}\right)\right], \\
M^{*}\left(\xi, t^{*}\right)=\frac{Q^{*}}{2} \xi(1-\xi), \\
F_{s}^{*}\left(\xi, t^{*}\right)=\frac{Q^{*}}{2}(1-2 \xi) .
\end{array}\right.
$$

To reveal the effect of viscosity, three kinds of cracked beam models are defined as follows: (1) The modeling of the viscoelastic beam and the equivalent viscoelastic rotational springs (VBVS); (2) the modeling of the viscoelastic beam and the equivalent elastic rotational springs (VBES); and (3) the modeling of the elastic beam and the equivalent elastic rotational springs (EBES). Suppose that the cracks are always opened, the parameters $\lambda_{2}, \lambda_{3}$, and $\lambda_{4 j}$ for three different kinds of cracked beam models are defined in Table 1.

\subsubsection{Bending of a Two-Span Continuous Beam with Open} Cracks under a Concentrated Sudden Load. Let us consider a simply supported continuous beam with two subspan lengths $L_{1}$ and $L_{2}$ subjected to a concentrated force $F_{0}$ at the location $x=x_{F}$ (Figure 3 ). There is an open crack with the depth $d_{1}$ in the first subspan $\left(x=x_{1}<L_{1}\right)$. Then, the loads acting on the beams are given by the concentrated force $F_{0} H(t)$ and the reaction $R(t)$ at the intermediate support, i.e.,

$$
\begin{aligned}
& q(x, t)=F_{0} H(t) \delta\left(x-x_{F}\right)-R(t) \delta\left(x-L_{1}\right), \\
& \bar{q}(x, s)=\frac{1}{s} F_{0} \delta\left(x-x_{F}\right)-\bar{R} \delta\left(x-L_{1}\right) .
\end{aligned}
$$

Let $L_{2}=L-L_{1}$, and $\left\{\begin{array}{l}\bar{q}^{[1]}(x, s)=\frac{1}{s} F_{0} H\left(x-x_{F}\right)-\bar{R} H\left(x-L_{1}\right), \\ \bar{q}^{[2]}(x, s)=\frac{1}{s} F_{0}\left(x-x_{F}\right) H\left(x-x_{F}\right)-\bar{R}\left(x-L_{1}\right) H\left(x-L_{1}\right), \\ \bar{q}^{[3]}(x, s)=\frac{1}{2 s} F_{0}\left(x-x_{F}\right)^{2} H\left(x-x_{F}\right)-\frac{1}{2} \bar{R}\left(x-L_{1}\right)^{2} H\left(x-L_{1}\right), \\ \bar{q}^{[4]}(x, s)=\frac{1}{6 s} F_{0}\left(x-x_{F}\right)^{3} H\left(x-x_{F}\right)-\frac{1}{6} \bar{R}\left(x-L_{1}\right)^{3} H\left(x-L_{1}\right) .\end{array}\right.$

Combining equations (26) and (27) and boundary conditions of equation (31), we obtains 
TABle 1: Parameters of the three kinds of cracked beam models with open cracks.

\begin{tabular}{lcccccc}
\hline Model & $Y(t)$ & $G(t)$ & $k_{j}(t)$ & $\lambda_{2}$ & $\lambda_{3}$ & $\lambda_{4 j}$ \\
\hline VBVS & $Y(t)$ & $\lambda_{1} Y(t)$ & $\mu_{j} Y(t) I$ & $\left(1 / q_{0}\right)+\left(p_{1}-\left(q_{1} / q_{0}\right)\right)\left(1 / q_{1}\right) e^{-\left(q_{0} / q_{1}\right) t}$ & $\lambda_{2} / \lambda_{1}$ & $\lambda_{2} /\left(I \mu_{j}\right)$ \\
VBES & $Y(t)$ & $\lambda_{1} Y(t)$ & $\mu_{j} E_{1} I$ & $\left(1 / q_{0}\right)+\left(p_{1}-\left(q_{1} / q_{0}\right)\right)\left(1 / q_{1}\right) e^{-\left(q_{0} / q_{1}\right) t}$ & $\lambda_{2} / \lambda_{1}$ & $1 /\left(\mu_{j} E_{1} I\right)$ \\
EBES & $E_{1}$ & $\lambda_{1} E_{1}$ & $\mu_{j} E_{1} I$ & $1 / E_{1}$ & $1 / \lambda_{1} E_{1}$ & $1 /\left(\mu_{j} E_{1} I\right)$ \\
\hline
\end{tabular}

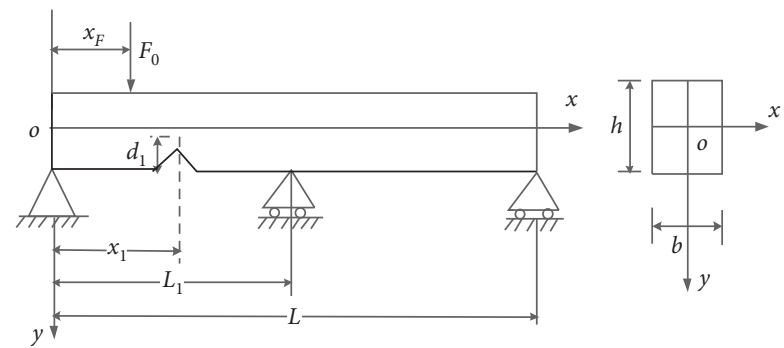

Figure 3: Geometric parameters of the continuous viscoelastic cracked beam.

$$
\left\{\begin{array}{l}
C_{1}=-\frac{1}{L}\left[\frac{1}{s}\left(L-x_{F}\right) F_{0}-L_{2} \bar{R}\right], \\
C_{2}=0, \\
C_{4}=0 \\
C_{3}=\left\{\frac{\left(L-x_{F}\right)}{\kappa A L s^{2} \bar{G}(s)}+\frac{x_{F}\left(L-x_{F}\right)\left(2 L-x_{F}\right)}{6 I L s^{2} \bar{Y}(s)}-\frac{1}{L^{2} s^{2} \bar{k}_{1}(s)}\left(L-x_{1}\right)=\left[L\left(x_{1}-x_{F}\right) H\left(x_{1}-x_{F}\right)-x_{1}\left(L-x_{F}\right)\right]\right\}, \\
F_{0}-\left\{\frac{L_{2}}{\kappa A L s \bar{G}(s)}+\frac{L_{1} L_{2}\left(2 L-L_{1}\right)}{6 I L s \bar{Y}(s)}-\frac{L-x_{1}}{L^{2} s \bar{k}_{1}(s)}\left[L\left(x_{1}-L_{1}\right) H\left(x_{1}-L_{1}\right)-L_{2} x_{1}\right]\right\} \bar{R} .
\end{array}\right.
$$

By substituting equation (39) into equation (26), and employing the deflection constraint condition $\bar{w}\left(L_{1}, s\right)=0$ at the intermediate support, we obtains

$$
\begin{aligned}
\bar{R}= & F_{0}\left\{\frac{1}{\kappa A s^{2} \bar{G}(s)}\left[\frac{L_{1}}{L}\left(L-x_{F}\right)-\left(L_{1}-x_{F}\right) H\left(L_{1}-x_{F}\right)\right]\right. \\
& +\frac{1}{6 I s^{2} \bar{Y}(s)}\left[\left(L_{1}-x_{F}\right)^{3} H\left(L_{1}-x_{F}\right)+\frac{L_{1}}{L}\left(L-x_{F}\right)\left(2 L x_{F}-x_{F}^{2}-L_{1}^{2}\right)\right] \\
& \left.+\frac{1}{s^{2} \bar{k}_{1}(s)}\left[\left(L_{1}-x_{1}\right) H\left(L_{1}-x_{1}\right)-\frac{L_{1}}{L}\left(L-x_{1}\right)\right]\left[\left(x_{1}-x_{F}\right) H\left(x_{1}-x_{F}\right)-\frac{\left.\left(L-x_{F}\right) x_{1}\right]}{L}\right]\right\} \\
& \times\left\{\frac{1}{s \bar{k}_{1}(s)}\left[\left(L_{1}-x_{1}\right) H\left(L_{1}-x_{1}\right)-\frac{\left(L-x_{1}\right) L_{1}}{L}\right]\left[\left(x_{1}-L_{1}\right) H\left(x_{1}-L_{1}\right)-\frac{L_{2} x_{1}}{L}\right]+\frac{L_{2} L_{1}}{\kappa L A s \bar{G}(s)}+\frac{L_{1}^{2} L_{2}^{2}}{3 L I s \bar{Y}(s)}\right\}^{-1} .
\end{aligned}
$$


Combining equations (28), (29), and (40), we get

$$
\bar{R}=\frac{1}{s} R_{0}
$$

where

$$
\begin{aligned}
R_{0}= & F_{0}\left\{\frac{1}{\lambda_{1} \kappa A}\left[\frac{L_{1}}{L}\left(L-x_{F}\right)-\left(L_{1}-x_{F}\right) H\left(L_{1}-x_{F}\right)\right]\right. \\
& +\frac{1}{6 I}\left[\left(L_{1}-x_{F}\right)^{3} H\left(L_{1}-x_{F}\right)+\frac{L_{1}}{L}\left(L-x_{F}\right)\left(2 L x_{F}-x_{F}^{2}-L_{1}^{2}\right)\right] \\
& \left.+\frac{1}{\mu_{1} I}\left[\left(L_{1}-x_{1}\right) H\left(L_{1}-x_{1}\right)-\frac{L_{1}}{L}\left(L-x_{1}\right)\right]\left[\left(x_{1}-x_{F}\right) H\left(x_{1}-x_{F}\right)-\frac{\left.\left(L-x_{F}\right) x_{1}\right]}{L}\right]\right\} \\
& \times\left\{\frac{1}{\mu_{1} I}\left[\left(L_{1}-x_{1}\right) H\left(L_{1}-x_{1}\right)-\frac{\left(L-x_{1}\right) L_{1}}{L}\right]\left[\left(x_{1}-L_{1}\right) H\left(x_{1}-L_{1}\right)-\frac{L_{2} x_{1}}{L}\right]+\frac{L_{2} L_{1}}{\lambda_{1} \kappa L A}+\frac{L_{1}^{2} L_{2}^{2}}{3 I L}\right\}^{-1} .
\end{aligned}
$$

By substituting equations (39) and (41) into equations (22), (26), and (27), introducing the dimensionless equation (35) and defining the dimensionless parameters by $\xi_{\mathrm{F}}=x_{\mathrm{F}} / L$, $L_{1}^{*}=L_{1} / L, L_{2}^{*}=L_{2} / L, F^{*}=F_{0} /\left(E_{1} L^{2}\right)$, and $R_{0}^{*}=R_{0} /\left(E_{1} L^{2}\right)$, the expressions for the dimensionless deflection, rotation angle, bending moment and shearing force of the continuous viscoelastic cracked beams can be expressed as, respectively

$$
\begin{aligned}
& w^{*}\left(\xi, t^{*}\right)=\frac{\lambda_{3}^{*} F^{*}}{\kappa A^{*}}\left[\left(1-\xi_{F}\right) \xi-\left(\xi-\xi_{F}\right) H\left(\xi-\xi_{F}\right)\right] \\
& +\frac{\lambda_{2}^{*} F^{*}}{6 I^{*}}\left[\left(\xi-\xi_{F}\right)^{3} H\left(\xi-\xi_{F}\right)+\left(1-\xi_{F}\right) \xi\left(2 \xi_{F}-\xi_{F}^{2}-\xi^{2}\right)\right] \\
& +\lambda_{41}^{*} F^{*}\left[\left(\xi-\xi_{1}\right) H\left(\xi-\xi_{1}\right)-\left(1-\xi_{1}\right) \xi\right]\left[\left(\xi_{1}-\xi_{F}\right) H\left(\xi_{1}-\xi_{F}\right)-\left(1-\xi_{F}\right) \xi_{1}\right] \\
& -\frac{\lambda_{3}^{*}}{\kappa A^{*}} R_{0}^{*}\left[L_{2}^{*} \xi-\left(\xi-L_{1}^{*}\right) H\left(\xi-L_{1}^{*}\right)\right] \\
& -\frac{\lambda_{2}^{*}}{6 I^{*}} R_{0}^{*}\left[\left(\xi-L_{1}^{*}\right)^{3} H\left(\xi-L_{1}^{*}\right)+L_{2}^{*} \xi\left(2 L_{1}^{*}-L_{1}^{* 2}-\xi^{2}\right)\right] \\
& -\lambda_{41}^{*} R_{0}^{*}\left[\left(\xi-L_{1}^{*}\right) H\left(\xi-L_{1}^{*}\right)-\left(1-\xi_{1}\right) \xi\right]\left[\left(\xi_{1}-L_{1}^{*}\right) H\left(\xi_{1}-L_{1}^{*}\right)-L_{2}^{*} \xi_{1}\right], \\
& \cdot \phi^{*}\left(\xi, t^{*}\right)=\frac{\lambda_{2}^{*}}{2 I^{*}} F^{*}\left[\left(\xi-\xi_{F}\right)^{2} H\left(\xi-\xi_{F}\right)+\frac{1}{3}\left(1-\xi_{F}\right)\left(2 \xi_{F}-\xi_{F}^{2}-3 \xi^{2}\right)\right] \\
& +\frac{\lambda_{2}^{*}}{\mu_{1}^{*} I} F^{*}\left[H\left(\xi-\xi_{1}\right)-\left(1-\xi_{1}\right)\right]\left[\left(\xi_{1}-\xi_{F}\right) H\left(\xi_{1}-\xi_{F}\right)-\left(1-\xi_{F}\right) \xi_{1}\right] \\
& -\frac{\lambda_{2}^{*}}{2 I^{*}} R_{0}^{*}\left[\left(\xi-L_{1}^{*}\right)^{2} H\left(\xi-L_{1}^{*}\right)+\frac{1}{3} L_{2}^{*}\left(2 L_{1}^{*}-L_{1}^{* 2}-3 \xi^{2}\right)\right] \\
& -\frac{\lambda_{2}^{*}}{\mu_{1}^{*} I} R_{0}^{*}\left[H\left(\xi-\xi_{1}\right)-\left(1-\xi_{1}\right)\right]\left[\left(\xi_{1}-L_{1}^{*}\right) H\left(\xi_{1}-L_{1}^{*}\right)-L_{2}^{*} \xi_{1}\right] \\
& \cdot M^{*}\left(\xi, t^{*}\right)=-F^{*}\left[\left(\xi-\xi_{F}\right) H\left(\xi-\xi_{F}\right)-\left(1-\xi_{F}\right) \xi\right]+R_{0}^{*}\left[\left(\xi-L_{1}^{*}\right) H\left(\xi-L_{1}^{*}\right)-L_{2}^{*} \xi\right], \\
& \cdot F_{s}^{*}\left(\xi, t^{*}\right)=-F^{*}\left[H\left(\xi-\xi_{F}\right)-\left(1-\xi_{F}\right)\right]+R_{0}^{*}\left[H\left(\xi-L_{1}^{*}\right)-L_{2}^{*}\right],
\end{aligned}
$$


where

$$
\begin{aligned}
R_{0}^{*}= & F^{*}\left\{\frac{1}{\lambda_{1} \kappa A^{*}}\left[\left(1-\xi_{F}\right) L_{1}^{*}-\left(L_{1}^{*}-\xi_{F}\right) H\left(L_{1}^{*}-\xi_{F}\right)\right]\right. \\
& +\frac{1}{6 I^{*}}\left[\left(L_{1}^{*}-\xi_{F}\right)^{3} H\left(L_{1}^{*}-\xi_{F}\right)+L_{1}^{*}\left(1-\xi_{F}\right)\left(2 \xi_{F}-\xi_{F}^{2}-L_{1}^{* 2}\right)\right] \\
& \left.+\frac{1}{\mu_{1}^{*} I}\left[\left(L_{1}^{*}-\xi_{1}\right) H\left(L_{1}^{*}-\xi_{1}\right)-\left(1-\xi_{1}\right) L_{1}^{*}\right]\left[\left(\xi_{1}-\xi_{F}\right) H\left(\xi_{1}-\xi_{F}\right)-\left(1-\xi_{F}\right) \xi_{1}\right]\right\} \\
& \times\left\{\frac{1}{\mu_{1}^{*} I}\left[\left(L_{1}^{*}-\xi_{1}\right) H\left(L_{1}^{*}-\xi_{1}\right)-\left(1-\xi_{1}\right) L_{1}^{*}\right]\left[\left(\xi_{1}-L_{1}^{*}\right) H\left(\xi_{1}-L_{1}^{*}\right)-L_{2}^{*} \xi_{1}\right]+\frac{L_{2}^{*} L_{1}^{*}}{\lambda_{1} \kappa A^{*}}+\frac{L_{1}^{*} L_{2}^{* 2}}{3 I^{*}}\right\}^{-1} .
\end{aligned}
$$

\section{Results and Discussion}

3.1. Bending Analysis of the Simply Supported Beam with an Open Crack. In the four-point bending test of the Douglas fir beams at the bottom of the beam, Yahyaei-Moayyed and Taheri [30] presented the relationship between stress and strain using the Norton's power law as follows:

$$
\varepsilon=\varepsilon_{0}+m \sigma^{k} t^{n}
$$

where $\varepsilon_{0}$ is the initial elastic strain depending on the stress level, it is defined as $\varepsilon_{0}=\sigma / E$, and the average value of the tensile elastic modulus is $E=14 \mathrm{GPa}$. Additionally, the parameters are given as $\sigma=50 \mathrm{MPa}, m=2.645 \times 10^{-6} \mathrm{MPa}^{-k} \mathrm{~h}^{-n}, k=1.0$ and $n=0.348$. However, the physical meanings of the relevant parameters in the Norton's power law formula are not clear. Therefore, in the present study, the standard linear solid constitutive equation (1) is utilized to describe the viscoelastic properties of Douglas fir beams with the corresponding parameters as $\eta=6.9 \times 10^{3} \mathrm{GPa} \cdot \mathrm{h}, \quad E_{2}=39.68 \mathrm{GPa}$, and $E_{1}=14 \mathrm{GPa}$, which are obtained by fitting with Origin software. The fitting curve is depicted by the least square method in Figure 4. It is seen that these parameters are reasonable to some degree, and the coefficient of determination is $R^{2}=0.9217$ (the fitting accuracy is higher when the coefficient $R^{2} \longrightarrow 1$ ).

Meanwhile, the material and geometric parameters of the rectangular cracked beam are length $L=1 \mathrm{~m}$, width $b=0.1 \mathrm{~m}$, the crack coordinate at the midspan of the beam $x_{1}=L / 2$, crack depth $\mathrm{d}_{1} / h=0.5$, and Poisson's ratio $\nu=0.3$. Additionally, the uniform sudden load $Q_{0}=10^{6} \mathrm{~N} \cdot \mathrm{m}^{-1}$, and the shear correction factor $\kappa=10(1+\nu) /(12+11 \nu)$. Then, the bending behaviors of the simply supported viscoelastic beam with an open crack are analyzed as follows.

By Laplace transform and the principle of virtual work, Chen [8] presented a finite element method to analyze the quasistatic analysis of viscoelastic intact beams. On this basis, the finite element method for the viscoelastic Timoshenko cracked beam with VBVS model is presented to compare with the analytical solutions of the static bending deformation proposed in Sections 3.1 and 3.2. The quadratic Lagrangian interpolation function is used for the shape function of the element, the number of nodes is $n=2$, and the coordinates of nodes are $x_{1}=0$ and $x_{2}=L$. The FEM program required are implemented in Matlab, and run in a PC, which configures: OS Windows 7, CPU: intel i7 4790, memory: DDR3 $16 \mathrm{~GB}$, and SSD $128 \mathrm{~GB}$. Due to the space limitation, the detailed process is omitted. Figures 5 and 6 show the distribution of dimensionless midspan deflection $w^{*}\left(0.5, t^{*}\right)$, dimensionless deflection and rotation angle of a viscoelastic cracked beam with VBVS model, respectively. It is seen that the results of the present analytical expressions equation (37) are in good agreement with those of the FEM program. In the computation, the beam is uniformly meshed by 100 elements. From the figures, it is observed that the dimensionless deflection of the viscoelastic cracked beam shows obviously creep property with the time increasing. Also, for the crack effect at the crack location $\xi_{1}=0.5$, there exist a cusp on the deflection curve and a jump on the rotation angle curve.

Figure 7 shows the effects of the cracked beam models on the response of dimensionless midspan deflection $w^{*}\left(0.5, t^{*}\right)$ versus dimensionless time $t^{*}$. It is seen that for an elastic beam and an equivalent elastic cracked spring, the dimensionless deflection of the EBES beam model is equal to a constant, which is independent with the time $t^{*}$. Although for the viscoelastic beams with the VBVS model and VBES model, the equivalent bending stiffness of the beam decreases with the time increasing, and the corresponding midspan deflection increases and tends to be a certain value gradually. The dimensionless deflection of the VBVS model is greater than that of the VBES model, which shows that the viscosity of the equivalent cracked spring has the significant influence on the beam bending deformation. The reason can be explained that considering that the VBVS model and VBES model have the viscoelastic rotational spring and elastic rotational spring, respectively, the equivalent bending stiffness of the VBVS model is less than that of the VBES model. In addition, when $t^{*}=0$, the relaxation modulus is $Y(0)=E_{1}$, it means that the VBVS model and VBES model are both degenerated into the EBES model, so the deflections of the three kinds of cracked beam models are equal to each other.

Let $L / h=10$, the distributions of the dimensionless deflection and rotation angle versus dimensionless coordinate $\xi$ of the viscoelastic cracked beam are given in Figure 8. It is 


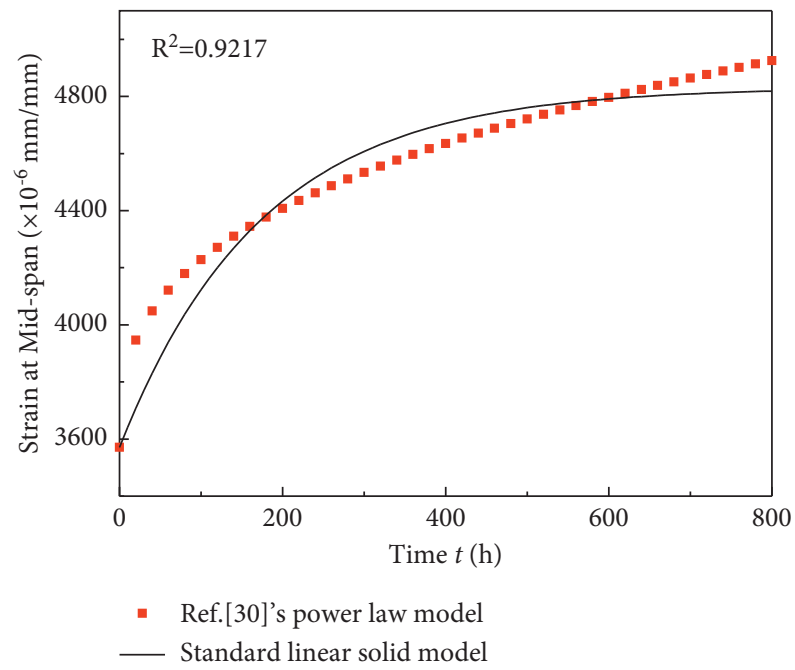

Figure 4: The fitting curve of the standard linear solid constitutive model.

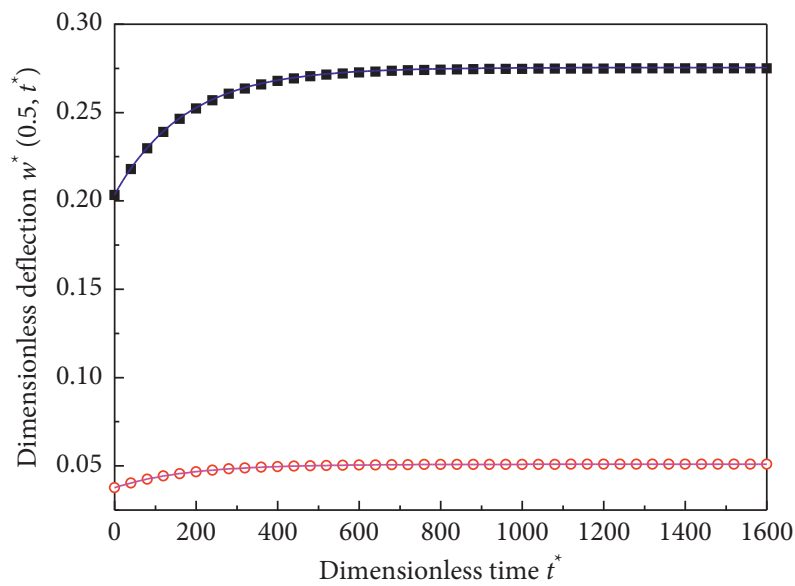

VBVS Model

- $L / h=10$, FEM's results $\quad L / h=10$, Exact results

- $L / h=5$, FEM's results $\quad L / h=5$, Exact results

FIGURE 5: Response of dimensionless midspan deflection $w^{*}\left(0.5, t^{*}\right)$ versus dimensionless time $t^{*}$ of viscoelastic cracked beam with VBVS model.

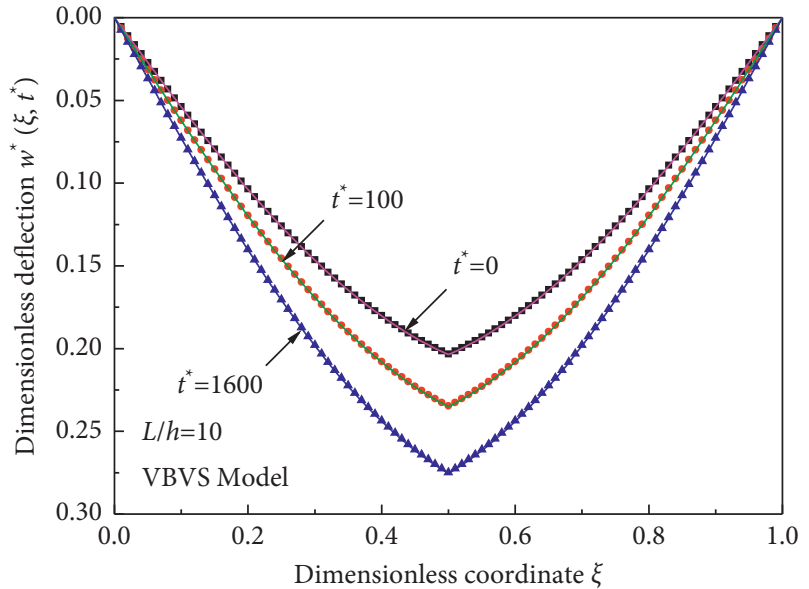

- FEM's results

— Exact results

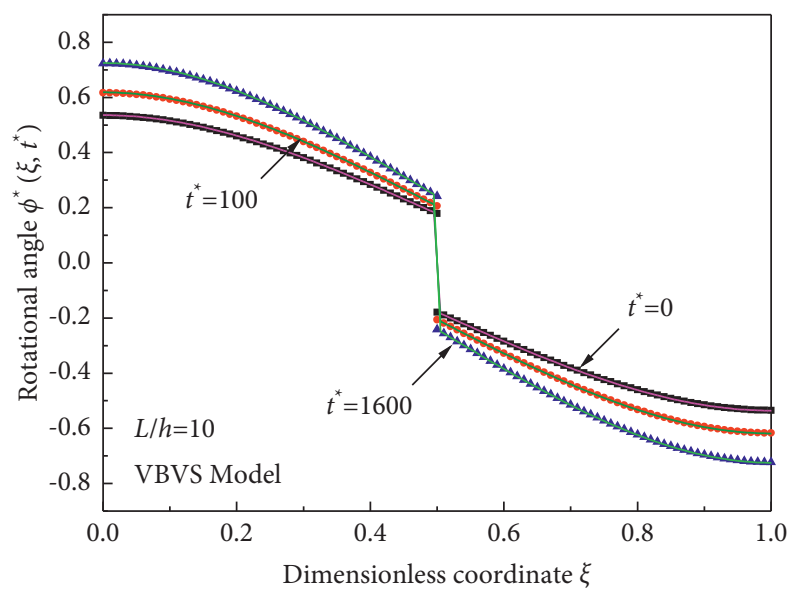

- FEM's results

— Exact results 


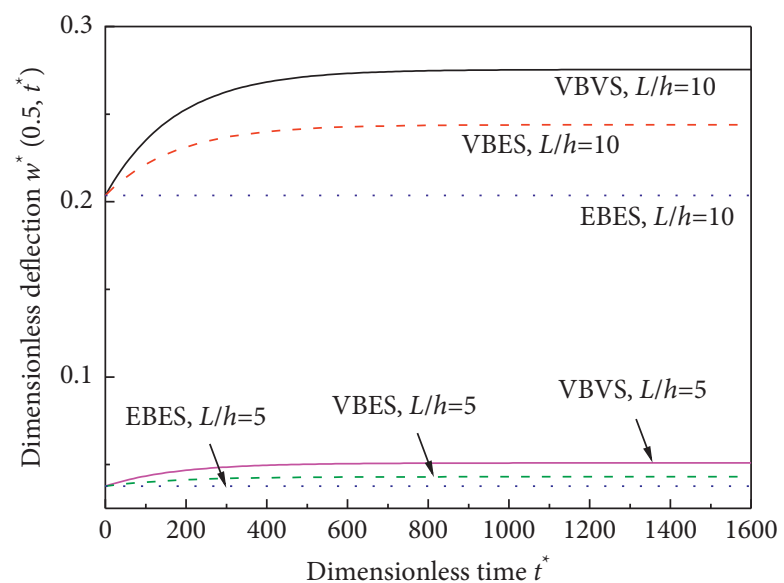

Figure 7: Response of dimensionless midspan deflection $w^{*}\left(0.5, t^{*}\right)$ versus dimensionless time $t^{*}$ of the viscoelastic beams with different cracked models.

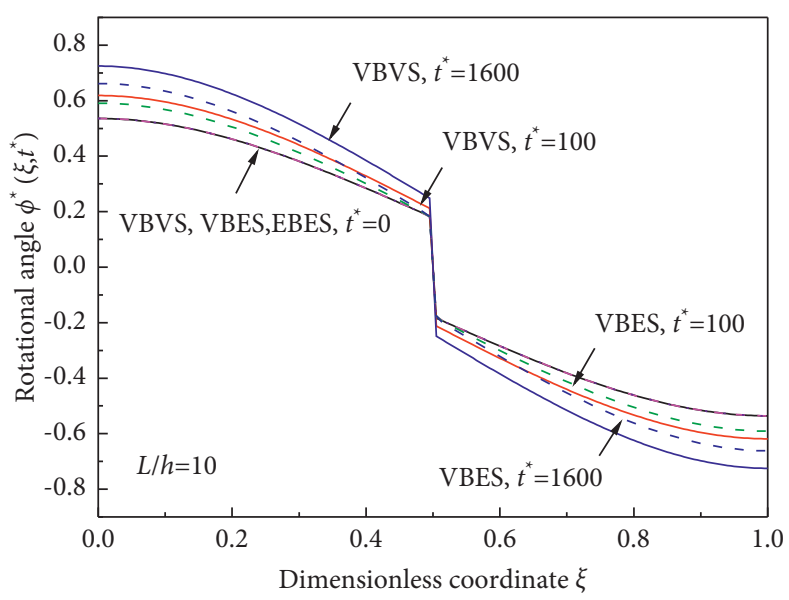

(a)

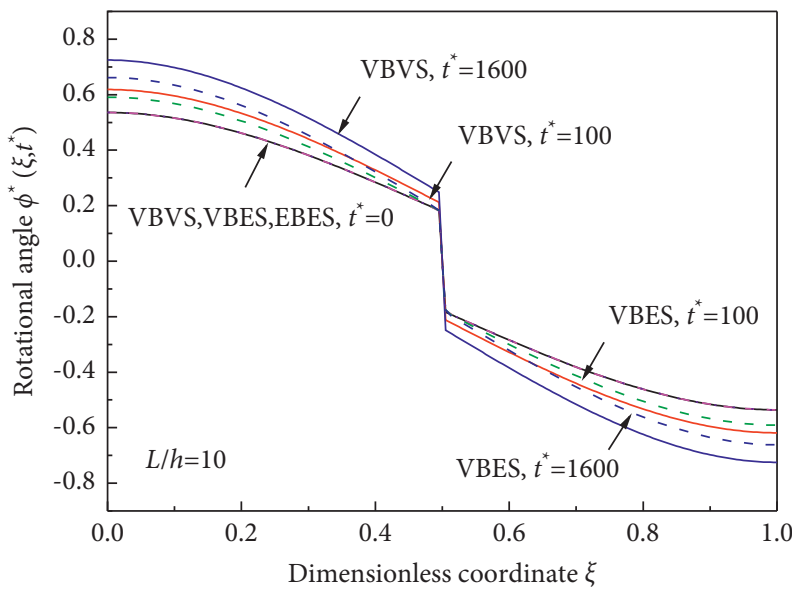

(b)

Figure 8: (a) Distribution of dimensionless deflection $w^{*}\left(\xi, t^{*}\right)$ versus dimensionless coordinate $\xi$ of the viscoelastic cracked beam with different cracked beam models. (b) Distribution of dimensionless rotational angle $\varphi^{*}\left(\xi, t^{*}\right)$ versus dimensionless coordinate $\xi$ of the viscoelastic cracked beam with different cracked beam models.

observed that for the three cracked beam models, there always has a cusp on the deflection curve and a jump on the rotation angle curve at the crack location $\xi_{1}=0.5$. For the VBVS and VBES beam models, the dimensionless midspan deflection $w^{*}\left(0.5, t^{*}\right)$ increases with dimensionless time. When $t^{*}=100$, the deflections $w^{*}\left(0.5, t^{*}\right)$ of the two beam models are 1.154 times and 1.087 times of that when $t^{*}=0$, respectively. When $t^{*}=1600$, they change to be 1.353 times and 1.198 times, respectively. However, the dimensionless deflection of the EBES beam model is a certain constant. Additionally, considering the effects of the viscoelastic beam and the equivalent viscoelastic rotational springs, the phenomenon of crack effect (deflection cusp and jump of rotation angle) for the VBVS model at the crack location $\xi_{1}=0.5$ is more significant, which reflects that the effects of viscosity cannot be ignored.

Let $\kappa \longrightarrow \infty$, equation (36) is degenerated into the bending expression of the viscoelastic Euler-Bernoulli cracked beam. When $t^{*}=1600$, the responses of dimensionless midspan deflection $w^{*}\left(0.5, t^{*}\right)$ of the Timoshenko and Euler-Bernoulli cracked beams are presented in Figure 9. Due to the shearing deformation caused by the shearing force, there is an additional deflection of the Timoshenko beam model. The difference between the midspan deflections of the Timoshenko and Euler-Bernoulli cracked beams is a constant for the VBVS model or VBES model, which can be interpreted by taking the deflection expression equation (36) as an example: it is clear that the difference in the deflections is depend on the parameter $\lambda_{3}^{*}$, which is related to the relaxation modulus and Poisson's ratio of the viscoelastic materials, but the slenderness ratio does notwork.

Let $L / h=10$ and $t^{*}=1600$, the effects of crack depth $d_{1} / h$ on the dimensionless midspan deflection $w^{*}\left(0.5, t^{*}\right)$ and deflection $w^{*}\left(\xi, t^{*}\right)$ are given in Figures 10 and 11, respectively. It is seen that, the dimensionless midspan deflection increases with the crack depth increasing, and for 


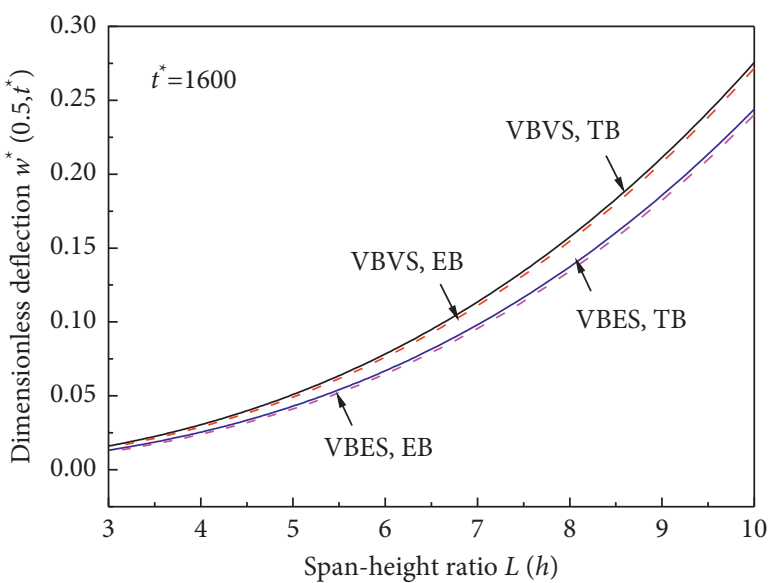

Figure 9: Response of dimensionless midspan deflection $w^{*}(0.5$, $\left.t^{*}\right)$ versus slenderness ratio $L / h$ of the viscoelastic Timoshenko and Euler cracked beams.

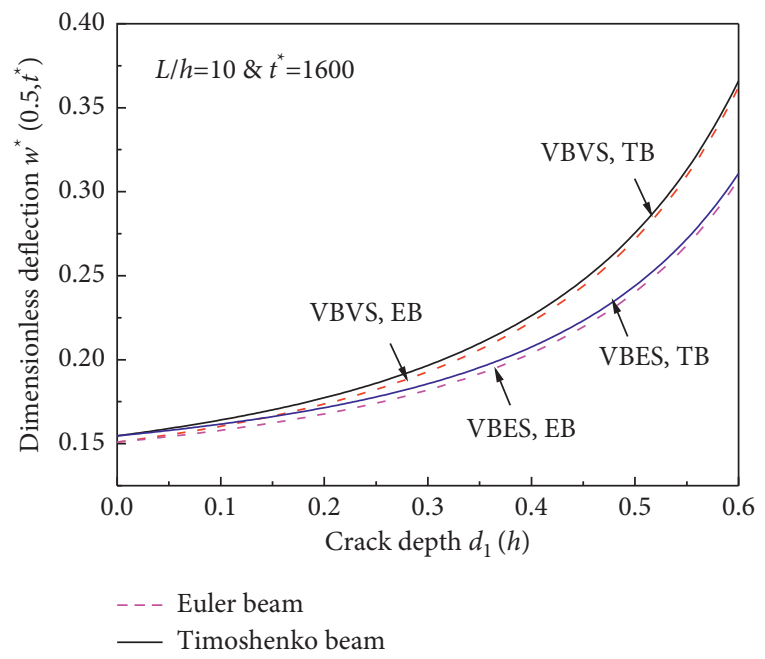

Figure 10: Response of dimensionless midspan deflection $w^{*}(0.5$, $\left.t^{*}\right)$ versus crack depth $d_{1} / h$ of viscoelastic Timoshenko and Euler cracked beams.

the VBVS model and VBES model, the difference of the dimensionless midspan deflection between the Timoshenko beam and Euler-Bernoulli beam is independent with the crack depth.

\subsection{Bending Analysis of the Continuous Beam with an Open} Crack. Consider that there exist a simply supported continuous beam with two subspan lengths $L_{1}=L_{2}=0.5 \mathrm{~m}$, subjected to a concentrated force at $x=x_{F}$, and an open crack is in the middle of the first span. The geometric and physical parameters are the same as those used in Section 3.1.

Let $x_{\mathrm{F}}=0.275 \mathrm{~m}, L / h=10$, and $d_{1} / h=0.5$, the dimensionless deflection of the viscoelastic Timoshenko cracked beam with VBVS model is obtained by equation (43). In Figure 12, the results calculated by equation (43) and the FEM programs are in good agreement with each other, which indicates that the correctness of the present model

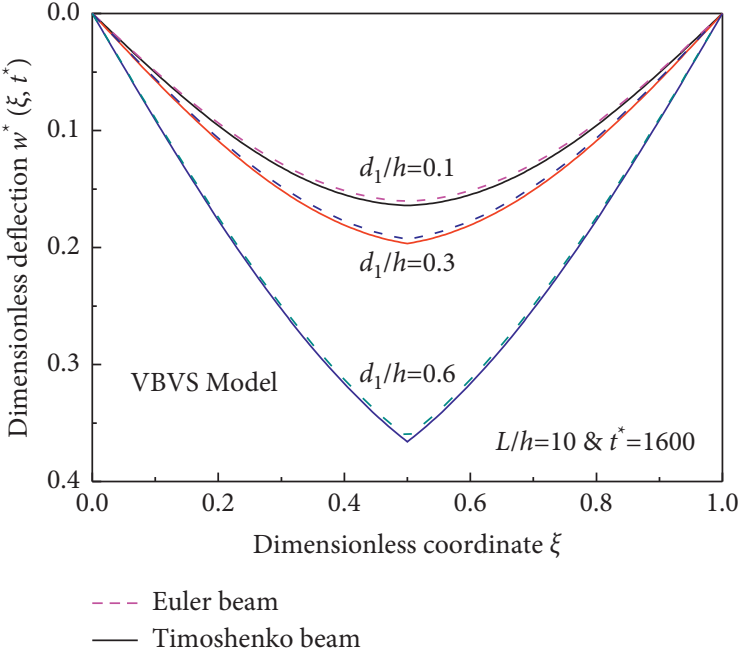

Figure 11: Distribution of dimensionless deflection $w^{*}\left(\xi, t^{*}\right)$ versus dimensionless coordinate $\xi$ of viscoelastic Timoshenko and Euler cracked beams with different crack depth $d_{1} / h$.

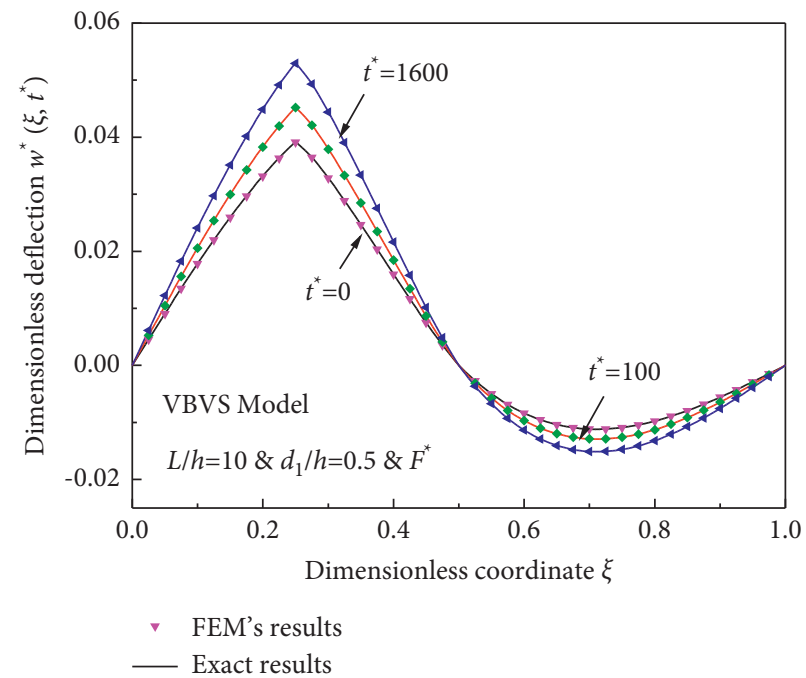

Figure 12: Distribution of dimensionless deflection $w^{*}\left(\xi, t^{*}\right)$ versus dimensionless coordinate $\xi$ of continuous viscoelastic cracked beam with VBVS model.

and programs are verified in some degree. It is stated that the deflection of the viscoelastic cracked beam shows the creep property with the time increasing. Also, the phenomenon of deflection cusp is observed at the crack location.

Let $\xi_{\mathrm{F}}=0.125$, Figure 13 illustrates the effect of the slenderness ratio on the dimensionless deflection $w^{*}\left(0.25, t^{*}\right)$ versus the time $t^{*}$. It is seen that the deflection $w^{*}\left(0.25, t^{*}\right)$ increases with the time and slenderness ratio increasing and tends to be a certain value gradually. The deflection of the VBVS beam model is greater than that of the VBES beam model for the equivalent viscoelastic spring of the crack. When $t^{*}=0$, the relaxation modulus is degenerated into $Y(t)=E_{1}$, and the deflection of VBVS model is the same with that of VBES model. 


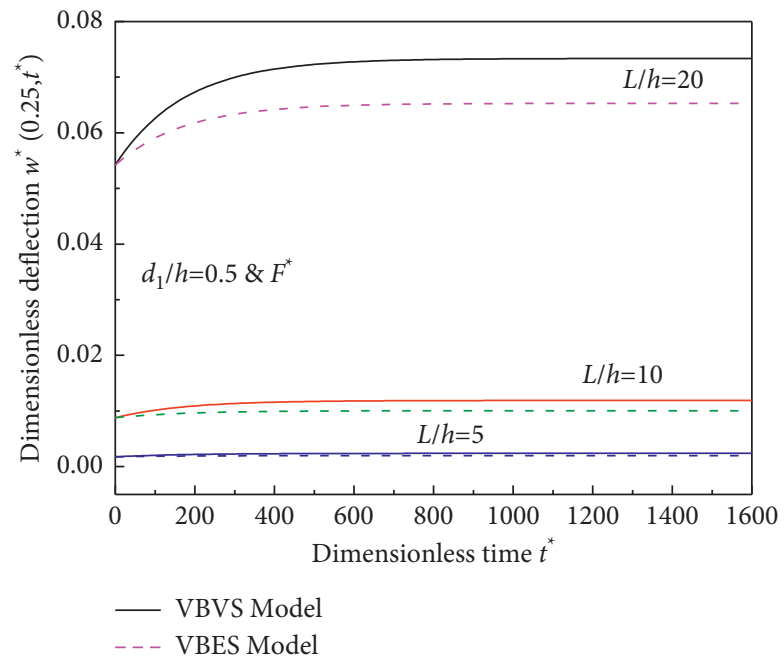

Figure 13: Response of dimensionless deflection $w^{*}\left(0.25, t^{*}\right)$ versus dimensionless time $t^{*}$ of the continuous cracked beams with different slenderness ratio $L / h$.

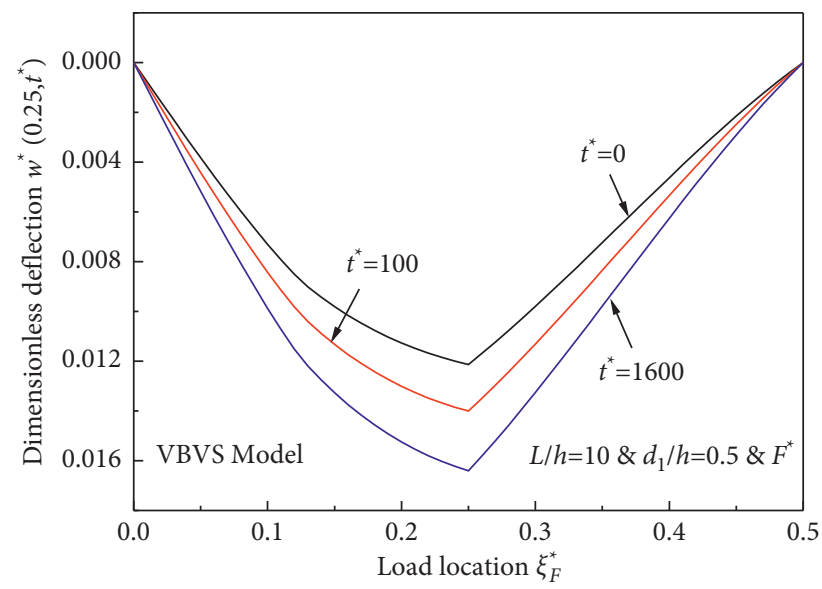

Figure 14: Response of dimensionless deflection $w^{*}\left(0.25, t^{*}\right)$ versus dimensionless load location coordinate $\xi_{\mathrm{F}}$ of the continuous cracked beams with different dimensionless time $t^{*}$.

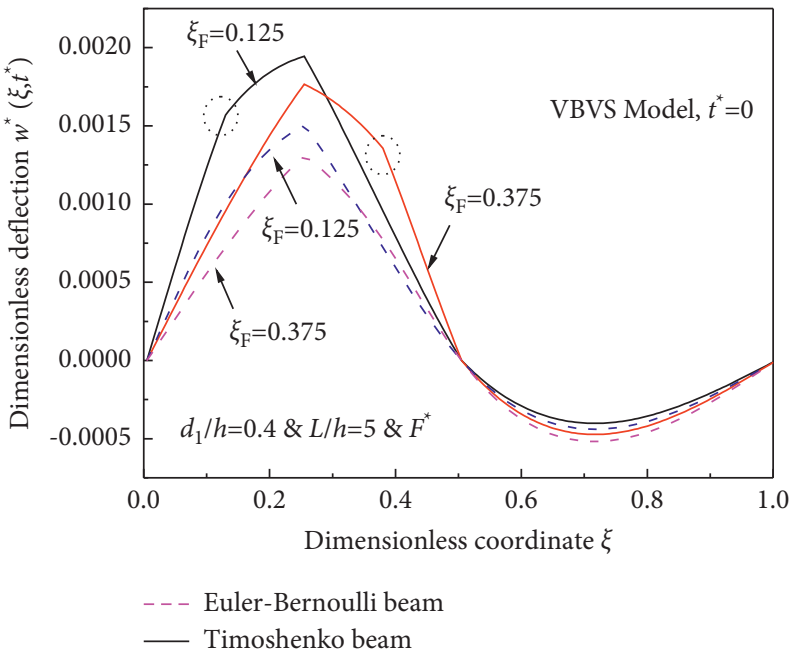

(a)

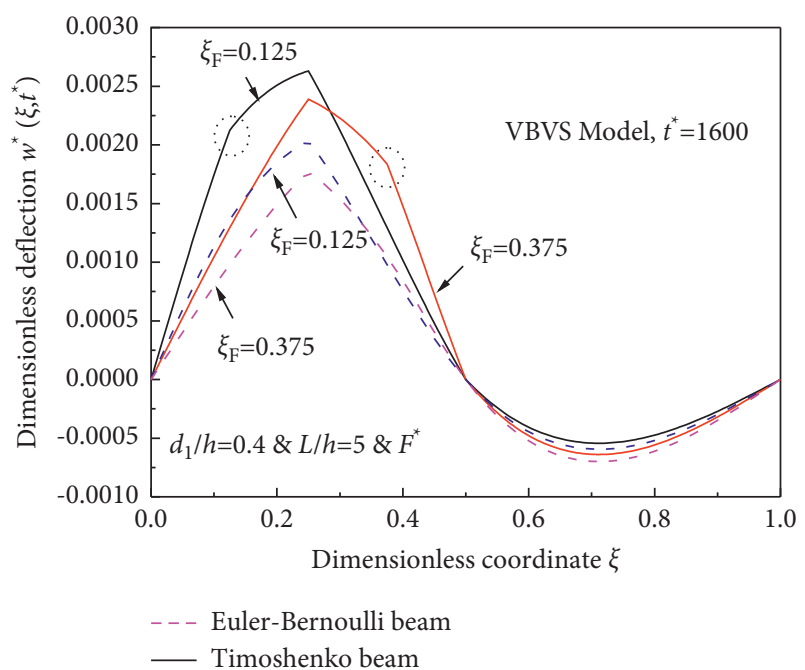

(b)

Figure 15: (a) Distribution of dimensionless deflection $w^{*}\left(\xi, t^{*}\right)$ versus dimensionless coordinate $\xi$ of the continuous cracked beams with VBVS model when $t^{*}=0$. (b) Distribution of dimensionless deflection $w^{*}\left(\xi, t^{*}\right)$ versus dimensionless coordinate $\xi$ of the continuous cracked beams with VBVS model when $t^{*}=1600$. 
Let $L / h=10$, Figure 14 shows the effects of the dimensionless time $t^{*}$ and load location $\xi_{F}$ on the dimensionless deflection $w^{*}\left(0.25, t^{*}\right)$ at the crack location. It is stated that when the concentrated load is applied at the first two support positions $\left(\xi_{F}=0\right.$ and 0.5$)$, the dimensionless deflection is zero, and the deflection reaches the maximum value as the concentrated load is applied at the crack location $\left(\xi_{F}=0.25\right)$.

Let $L / h=5, d_{1} / h=0.4$ and $\xi_{F}=0.125$ (or $\xi_{F}=0.375$ ), Figure 15 shows the dimensionless deflections $w^{*}\left(\xi, t^{*}\right)$ of the viscoelastic Timoshenko and Euler-Bernoulli cracked beams with VBVS model. Due to the effect of the open crack, there exists a deflection cusp at the crack location $\xi_{1}=0.25$. Meanwhile, the phenomenon of deflection cusp is also observed at the load location; it is because that the transversal shearing force of the beam is discontinuous at the load location $\xi_{F}$, and the shearing deformation caused by the shearing force induces the additional deflection in the Timoshenko cracked beam. However, for the Euler-Bernoulli cracked beam, there is no shear deformation, which means that no additional cusp exists on its deflection curve at the load location.

\section{Conclusions}

Regarding the transversal crack as a massless viscoelastic rotational spring, the equivalent bending stiffness of the viscoelastic cracked beam with open cracks are presented in Laplace domain using the generalized Dirac delta function and Laplace transform. Then, the generally explicit analytical expressions in time domain for the deflection and rotation angle of the Timoshenko beam having an arbitrary number of open cracks and satisfying the standard linear solid constitutive equation of viscoelasticity are derived. In numerical examples, the accuracy and applicability of the present analytical expressions and the finite element method are compared to be verified. Also, the effects of the time, slenderness ratio, and crack depth on the bending deformation under the simply supported boundary conditions with the different cracked beam models are analyzed, and the main findings are listed as follows:

(1) For a simply supported viscoelastic cracked beam subjected to a uniform sudden load, there exist a cusp on the deflection curve and a jump on the rotation angle curve at the crack location. In addition, compared with the bending deformations of the three kinds of cracked beam models, it reveals that the effects of the viscoelastic beam and equivalent viscoelastic rotational spring on the bending deflection curves are significant.

(2) For the VBVS model and VBES model, the dimensionless midspan deflection of the Timoshenko cracked beam is greater than that of the Euler-Bernoulli beam as the additional deformation induced by the transverse shearing force. Also, the difference of the deflection is dependent on the relaxation modulus and Poisson's ratio, whereas independent with the slenderness ratio, crack depth, or the cracked beam model.
(3) For the viscoelastic continuous cracked beams subjected to a concentrated sudden load, the dimensionless deflections of the Timoshenko and Euler-Bernoulli beams both have the deflection cusp at the crack location. In addition, due to the discontinuity of the shearing force at the load location, there also exists the phenomenon of deflection cusp at the load location for the Timoshenko beams, whereas no similar phenomenon is observed for the Euler-Bernoulli beams.

\section{Data Availability}

The data used to support the findings of this study are available from the corresponding author on request.

\section{Conflicts of Interest}

The authors declare that they have no conflicts of interest.

\section{Acknowledgments}

The authors acknowledge the financial support of Nanhu Scholars Program for Young Scholars of XYNU.

\section{References}

[1] D. R. Bland, The Theory of Linear Viscoelasticity, Pergamon Press, New York, NY, USA, 1960.

[2] R. M. Christensen, Theory of Viscoelasticity: An Introduction, Academic Press, New York, NY, USA, 2nd edition, 1982.

[3] K. Lee, "Large deflection of viscoelastic fiber beams," Textile Research Journal, vol. 77, no. 1, pp. 47-51, 2007.

[4] M. Vrable, T. Ionut-Ovidiu, and T. Jerca, "Differential equation of a visco-elastic beam subjected to bending," Bulletin of the Polytechnic Institute of Jassy Constructions Architecture, vol. 55, no. 2, pp. 21-32, 2009.

[5] S. Dost and P. G. Glockner, "On the dynamic stability of viscoelastic perfect columns," International Journal of Solids and Structures, vol. 18, no. 7, pp. 587-596, 1982.

[6] U. S. Shirahatti and S. C. Sinha, "On the stability of perfect viscoelastic columns," Journal of Sound and Vibration, vol. 174, no. 1, pp. 57-68, 1994.

[7] K. K. Stevens, "On the parametric excitation of a viscoelastic column," American Institute of Aeronautics and Astronautics Journal, vol. 4, no. 12, pp. 2111-2116, 2015.

[8] T.-M. Chen, "The hybrid Laplace transform/finite element method applied to the quasi-static and dynamic analysis of viscoelastic Timoshenko beams," International Journal for Numerical Methods in Engineering, vol. 38, no. 3, pp. 509-522, 1995.

[9] O. Martin, "A modified variational iteration method for the analysis of viscoelastic beams," Applied Mathematical Modelling, vol. 40, no. 17-18, pp. 7988-7995, 2016.

[10] M. I. Friswell and J. E. T. Penny, "Crack modeling for structural health monitoring," Structural Health Monitoring, vol. 1, no. 2, pp. 139-148, 2002.

[11] Q. Lyu, J. Li, and N. H. Zhang, "Quasi-static and dynamical analyses of a thermoviscoelastic Timoshenko beam using the differential quadrature method," Applied Mathematics and Mechanics, vol. 40, no. 4, pp. 131-144, 2018.

[12] Q. Lyu, N. H. Zhang, C. Y. Zhang, J. Z. Wu, and Y. C. Zhang, "Effect of adsorbate viscoelasticity on dynamical responses of 
laminated microcantilever resonators," Composite Structures, vol. 250, p. 10, Article ID 112553, 2020.

[13] L. Li and Y. Hu, "State-space method for viscoelastic systems involving general damping model," AIAA Journal, vol. 54, no. 10 , pp. $3290-3295,2016$.

[14] Z. Ding, L. Li, and Y. Hu, "A modified precise integration method for transient dynamic analysis in structural systems with multiple damping models," Mechanical Systems and Signal Processing, vol. 98, pp. 613-633, 2018.

[15] S. Caddemi, I. Caliò, and M. Marletta, "The non-linear dynamic response of the Euler-Bernoulli beam with an arbitrary number of switching cracks," International Journal of Nonlinear Mechanics, vol. 45, no. 7, pp. 714-726, 2010.

[16] B. Biondi and S. Caddemi, "Euler-Bernoulli beams with multiple singularities in the flexural stiffness," European Journal of Mechanics - A: Solids, vol. 26, no. 5, pp. 789-809, 2007.

[17] G. Buda and S. Caddemi, "Identification of concentrated damages in Euler-Bernoulli beams under static loads," Journal of Engineering Mechanics, vol. 133, no. 8, pp. 942-956, 2007.

[18] S. Caddemi and I. Caliò', "Exact solution of the multi-cracked Euler-Bernoulli column," International Journal of Solids and Structures, vol. 45, no. 5, pp. 1332-1351, 2008.

[19] A. Palmeri and A. Cicirello, "Physically-based Dirac's delta functions in the static analysis of multi-cracked EulerBernoulli and Timoshenko beams," International Journal of Solids and Structures, vol. 48, no. 14-15, pp. 2184-2195, 2011.

[20] A. Cicirello and A. Palmeri, "Static analysis of Euler-Bernoulli beams with multiple unilateral cracks under combined axial and transverse loads," International Journal of Solids and Structures, vol. 51, no. 5, pp. 1020-1029, 2014.

[21] X. Yang, J. Huang, and Y. Ouyang, "Bending of Timoshenko beam with effect of crack gap based on equivalent spring model," Applied Mathematics and Mechanics, vol. 37, no. 4, pp. 513-528, 2016.

[22] M. Donà, A. Palmeri, M. Lombardo, and A. Cicirello, "An efficient two-node finite element formulation of multi-damaged beams including shear deformation and rotatory inertia," Computers \& Structures, vol. 147, pp. 96-106, 2015.

[23] K. K. Stevens and R. M. Evan-Iwanowski, "Parametric resonance of viscoelastic columns," International Journal of Solids and Structures, vol. 5, no. 7, pp. 755-765, 1969.

[24] W. Szyszkowski and P. G. Glockner, "The imperfect linearly viscoelastic column," International Journal of Engineering Science, vol. 23, no. 10, pp. 1113-1120, 1985.

[25] D. Touati and G. Cederbaum, "Effects of compressibility on the postbuckling behavior of imperfect viscoelastic columns," Computers \& Structures, vol. 68, no. 6, pp. 561-566, 1998.

[26] H. H. Zhang, G. Rong, and L. X. Li, "Numerical study on deformations in a cracked viscoelastic body with the extended finite element method," Engineering Analysis with Boundary Elements, vol. 34, no. 6, pp. 619-624, 2010.

[27] D. Younesian, S. R. Marjani, and E. Esmailzadeh, "Nonlinear vibration analysis of harmonically excited cracked beams on viscoelastic foundations," Nonlinear Dynamics, vol. 71, no. 1-2, pp. 109-120, 2013.

[28] V. Sarvestan, H. R. Mirdamadi, M. Ghayour, and A. Mokhtari, "Spectral finite element for vibration analysis of cracked viscoelastic Euler-Bernoulli beam subjected to moving load," Acta Mechanica, vol. 226, no. 12, pp. 4259-4280, 2015.

[29] T. Q. Yang, Viscoelastic Theory and Application, Science Press, Beijing, China, 2004.
[30] M. Yahyaei-Moayyed and F. Taheri, "Experimental and computational investigations into creep response of AFRP reinforced timber beams," Composite Structures, vol. 93, no. 2, pp. 616-628, 2011. 